\title{
Effect of Cervical Mobilization, Transcutaneous Electrical Stimulation and Suboccipital Release in Cervicogenic Headache: A Case Report
}

\author{
Darshana Fursule $^{1}$, Kapil Garg ${ }^{2}$ \\ ${ }^{1}$ Masters in Musculoskeletal Physiotherapy (MPT-II), MGM Institute of Physiotherapy, Aurangabad \\ ${ }^{2}$ Associate Professor, Department of Musculoskeletal Physiotherapy, MGM Institute of Physiotherapy, \\ Aurangabad \\ Corresponding Author: Darshana Fursule
}

\begin{abstract}
Background: Cervicogenic headaches are one of the common musculoskeletal disorders that originates in the neck and are radiates from neck to head. Cervicogenic headache is usually treated with a comprehensive strategy that includes pharmacologic, nonpharmacologic, manipulative, anesthetic, surgical procedures and physiotherapy. Physiotherapy includes spinal manipulation, mobilization, myofascial release, exercises and electrotherapeutic modalities.

Purpose: This case report aims to evaluate the effects of combination of cervical spine mobilization, TENS, Suboccipital release on cervicogenic headache in maintaining long-term benefits.

Methods: This a case report of 31-year-old male having cervicogenic headache from 4-5 years. He reported symptoms like pain in right side neck which radiates into head till forehead and stiffness in neck. Physiotherapy treatment like cervical spine mobilization, TENS and Suboccipital release was given for 5 sessions (alternate days) for 10 days.

Outcome Measures: Numerical pain rating scale, Neck disability Index and Cervical range of motion was used as outcome measures.

Results: There was marked decrease in pain intensity from 8 on NPRS to 1 on NPRS. Also there was increase in cervical range of motion specially flexion, lateral flexion and rotation and decrease in neck disability index from 28 to 10 .

Conclusion: Physical therapy with TENS, Cervical spine mobilization \& sub occipital release can be used as an effective intervention protocol for reducing pain and stiffness and increasing range of motion in patients with Cervicogenic Headache.
\end{abstract}

Keywords: Transcutaneous electrical nerve stimulation, Cervical Spine mobilization, Suboccipital release, Cervicogenic Headache, Numerical Pain Rating Scale, Neck Disability Index

\section{INTRODUCTION}

Cervicogenic headaches are one of the common musculoskeletal disorders that originates in the neck and are radiates from neck to head. Cervicogenic headaches are classified as secondary headaches since they are caused by a secondary ailment such as neck injuries, strains, sprains, or infections. They differ from primary headaches like migraines and cluster headaches because of this. ${ }^{1}$ It's frequently accompanied by a decrease in neck range of motion (ROM). Cervicogenic headache is a rare chronic headache that affects adults aged 30 to 44 . Its prevalence ranges from $1 \%$ to $4 \%$ among headache sufferers. Males and females are affected in about equal amounts, with an $\mathrm{F} / \mathrm{M}$ ratio of 0.97 . The early $30 \mathrm{~s}$ are estimated to be the onset age. ${ }^{2}$

Pain begins in the neck (Ligamentum Nuchae, spinous process of cervical vertebra) and back of the head 
(Occipital region) in cervicogenic headaches and radiates to the front of the head (Frontal Region). Individuals, consultants, and physical therapists typically struggle to distinguish cervicogenic headaches from migraines and tension headaches since both induce neck pain. ${ }^{3}$ Cervicogenic headaches are characterized by a headache that is accompanied by neck pain and stiffness. Cervicogenic headaches affect one side of the face, the back of the head, and the neck in $90 \% .{ }^{4}$ Trigger points are most commonly located in the suboccipital, cervical, and shoulder muscles, and they can send pain to the head. Patients may experience scalp paresthesia or dysesthesia on occasion. ${ }^{5}$

Cervicogenic headache is usually treated with a comprehensive strategy that includes pharmacologic, nonpharmacologic, manipulative, anaesthetic, and surgical procedures. Nerve blocks can temporarily reduce pain and make physical therapy more effective. Physical therapy techniques such as stretches, exercises, and spinal manipulations, as well as other techniques such as deep breathing exercises, Yoga, and acupuncture, have been found to be effective in treating cervicogenic headaches. ${ }^{3}$ Physical therapy is an important part of cervicogenic headache treatment. Physical therapy is used as a first-line treatment option, along with pain relievers. The therapist has a critical role to play in addressing these symptoms and should make every effort to find appropriate treatments. Physiotherapy includes spinal manipulation, mobilization, myofascial release, exercises and electrotherapeutic modalities. ${ }^{3}$

Cervical Spine Mobilization is commonly used to improve neck range of motion and muscle balance between flexors and extensors, as well as between right and left side flexors. ${ }^{4}$ Myofascial Release MFR is a therapeutic technique that involves applying moderate pressure and stretching (in both direct and indirect approaches) with the goal of reducing pain, optimizing length, and facilitating the release of fascial constraints caused by injury, stress, repetitive use, and other factors. ${ }^{5}$ TENS is beneficial to patients who suffer from cervicogenic headaches. TENS is a technique that involves placing small electrodes on the skin and sending modest electrical signals to stimulate nerves near the source of pain. ${ }^{6}$

This case study focuses on supporting evidence-based treatment in the management of cervicogenic headache using TENS, cervical spine mobilization, and suboccipital release.

\section{CASE STUDY}

A 31-year-old male, pre-diagnosed case of right side cervicogenic headache was referred by orthopedist to physiotherapy department. He gave the history of severe right sided headache from last 4-5 years. He had taken medications and alternate therapies for it. But the patient was not regular in his medications and therapies, so, he didn't get relief from suboccipital pain and headache. His pain was sudden in onset and he had episodic pain which lasted for few hours to few days. He had been referred to physiotherapy OPD with chief complaint of severe neck pain which radiated into right side head and forehead region, along with restricted neck range of motion.

On pain evaluation, the intensity of pain was 8 on Numerical pain rating scale (NPRS) and the area of current symptoms were right sided suboccipital region, neck region and right-side head and forehead region. Neck movements aggravated the pain. No past medical or surgical history. On palpation there was tenderness present in suboccipital muscle of the right side. On examination there were trigger points present in suboccipital area of the right side. On assessing the cervical range of motion, there was restriction in flexion, left side flexion and lift side rotation. The passive intervertebral movements were hypomobile of right side. In addition to above assessment, the Neck Disability Index (NDI) was used to identify the patient's disability level in a more standardized 
manner. This patient's score was 28 out of a possible 50, which indicates moderate disability.

The short-term goal was to reduce pain and the long-term goals were to reduce disability, and improve function for the patient. Procedure of evaluation and treatment was explained in detail and a written informed consent was obtained from the patient.

Table 1: NPRS and NDI scores

\begin{tabular}{|l|l|l|}
\multicolumn{1}{l}{ Table 1: NPRS and NDI scores } \\
\hline & Pre treatment & Post treatment \\
\hline NPRS & 8 & 2 \\
\hline NDI Score & 28 & 10 \\
\hline
\end{tabular}

Table 2: Cervical range of motion

\begin{tabular}{|l|l|l|}
\hline Cervical ROM & Pre treatment & Post treatment \\
\hline Flexion & 38 & 50 \\
\hline Extension & 46 & 58 \\
\hline Rotation right side & 40 & 64 \\
\hline Rotation left side & 49 & 70 \\
\hline Lateral flexion right side & 34 & 50 \\
\hline Lateral flexion left side & 30 & 44 \\
\hline
\end{tabular}

\section{Outcome Measures:}

Intensity of pain, range of motion and disability was assessed using Numerical Pain Rating Scale, Goniometer and Neck Disability Index respectively. Outcome measures were evaluated pre and post treatment.

\section{Suboccipital release:}

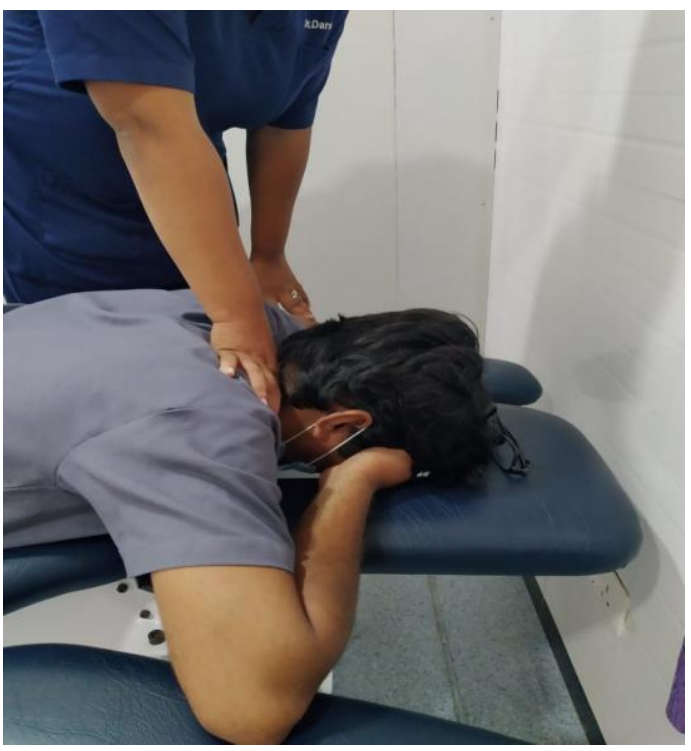

Fig 1. Cervical mobilization

The patient lied down in supine position with knee flexion and two or three

\section{PHYSIOTHERAPY INTERVENTION:}

Physical therapy with cervical spine Mobilizations, TENS and suboccipital release was carried out for 5 session alternate days for 10 days i.e., 3 sessions in $1^{\text {st }}$ week and 2 sessions in $2^{\text {nd }}$ week.

\section{Cervical Spine Mobilization:}

Central and unilateral posteroanterior pressures (Maitland) were applied to cervical vertebrae for reducing stiffness and improving range of motion. Patient was in prone position and therapist standing beside the patient. PA glide was applied at each cervical vertebrae spinous process and right zygapophyseal joints. Duration of Cervical spine Mobilization is 5 minutes. ${ }^{7}$

\section{Transcutaneous electrical stimulation TENS:}

Transcutaneous Electrical Nerve Stimulator Biotech system-two channeled were used. The daily treatment session lasted 10 minutes. Current frequency was $100 \mathrm{~Hz}$, and the wave duration was $50 \mathrm{msec}$. The power was adjusted to cause a tingling sensation. The stimulator was placed on paravertebral, suboccipital region bilaterally. ${ }^{10}$

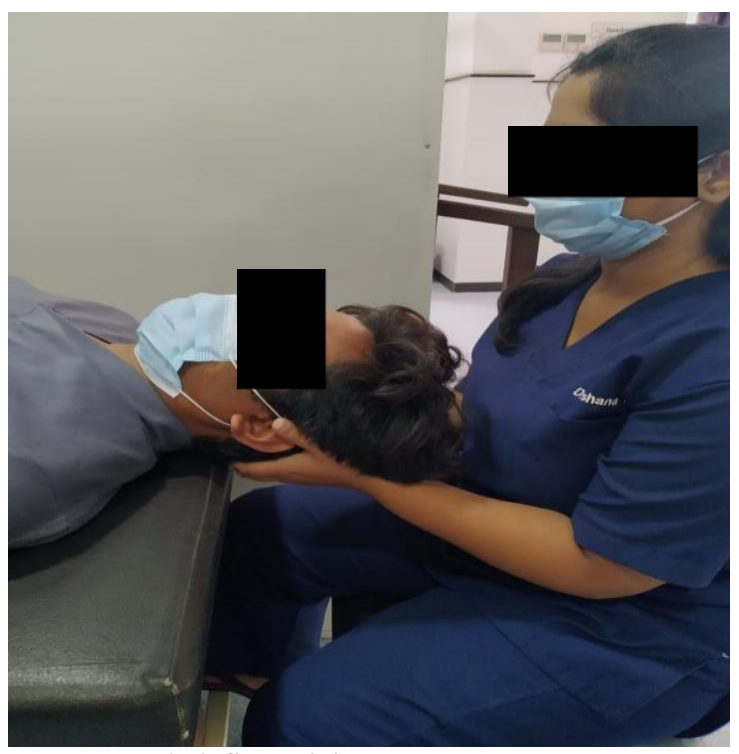

Fig 2. Suboccipital release

rolls of towel were placed under the head of the subjects to level the trunk with head. 
Therapist sat on a stool at the head of the table. Elbows and supinated forearms were on the table. The patient was asked to lift their head off the table. The tips of the first three fingers positioned into the soft tissue inferior to the arc of atlas. The fingers were stabilized in a flexed position -about $45^{\circ}$ at the MP and Proximal Interphalangeal (PIP) joints. The subjects were asked to rest their head back down so that their fingertips are over the suboccipital soft tissues and their finger pads rest tightly against the inferior aspect of the atlas. The procedure was done for 2 to 3 minutes. ${ }^{11}$

\section{RESULTS}

We found marked decrease in pain intensity from 8 on NPRS to 5 on NPRS immediately after $1^{\text {st }}$ session. Later when post intervention evaluation was done after 5 sessions, pain decreased to 1 on NPRS and there was increase in cervical range of motion specially flexion, lateral flexion and rotation and decrease in neck disability index from 28 to 10.

\section{DISCUSSION}

There are very few articles which has addressed the combined effects of cervical spine mobilization, suboccipital release and TENS in treating patients with cervicogenic headache. In a 30-year-old male, improvements in terms of pain reduction, range of motion, and functional capabilities were obtained. This case study demonstrated that this structured therapy regimen is extremely effective. There have been numerous studies on the efficiency of TENS alone, as well as TENS combined with Manipulations and Mobilizations, in various outcome measures. Many comparative studies have been done between Manipulations and Mobilizations, and both have been determined to be equally effective. ${ }^{4,6,7}$ Also, many studies have been done on the effectiveness of suboccipital release alone or in combination of cervical manipulation. ${ }^{9,11}$

Sub-occipital myofascial release works by lengthening and softening the connective tissue, creating permanent threedimensional length and width. ${ }^{5}$ MFR works by relaxing tensed muscles, boosting circulation, and triggering muscle and fascia stretch reflexes. This improves soft tissue extensibility, reducing pain and increasing range of motion. ${ }^{9}$ The goal of cervical manipulation is to release the fibrous adhesion that forms in the neck's zygapophyseal joints. Adhesion arises in the joints as a result of muscle stiffness. The joint becomes hypomobile as a result, and discomfort intensifies. When manipulation is used on the occipitoatlantal joint, the adhesion is broken and the facet joint opens up. It relieves pain by releasing muscle tension. ${ }^{4,8}$

TENS is thought to activate the inhibition of interneurons in the substantia gelatinosa of the posterior horn of the spinal cord, particularly big diameter fibres (Abeta), which prevent small diameter fibres from transmitting stimuli. The other theory claims that nerve stimulation causes the release of endorphins, which have been shown to have analgesic properties. ${ }^{6}$

In the patient we have found that Cervical spine mobilizations, sub occipital myofascial release, and TENS treatment showed significant reduction in pain and stiffness and restoring normal cervical range of motion. Despite the fact that this therapy protocol is not widely utilized, its relative effectiveness and benefits will undoubtedly benefit patients with cervicogenic headache of all ages, and it can be strongly advised in patients.

\section{CONCLUSION}

In our study we have found that the combine use of cervical mobilization, TENS and suboccipital release is effective in reduction of pain and disability and improving range of motion in patient with cervicogenic headache. TENS reduces pain, mobilizations increase range of motion, and suboccipital release reduces pain and increases soft tissue extensibility.

\section{Acknowledgement: None}


Darshana Fursule et.al. Effect of cervical mobilization, transcutaneous electrical stimulation and suboccipital release in cervicogenic headache: a case report.

\section{Conflict of Interest: None}

\section{Source of Funding: None}

\section{REFERENCES}

1. Page P. Cervicogenic headaches: an evidence-led approach to clinical management. Int J Sports Phys Ther. 2011 Sep; 6(3): 254-266.

2. Khalili Y., Murphy P. Cervicogenic Headache. StatPearls Publication; 2021 March 8.

3. Biondi D. Cervicogenic Headache: A Review of Diagnostic and Treatment Strategies. Journal of the American Osteopathic Association. 2005 April; 105(4): S16-S22.

4. Garcia J., Arnold S., Tetley K., Voight K., et al. Mobilization and Manipulation of the Cervical Spine in Patients with Cervicogenic Headache: Any Scientific Evidence? Front Neurol. 2016; 7: 40.

5. Arab A., Rameani E. Sub occipital myofascial release technique for the treatment of cervicogenic headache. Journal of Bodywork and Movement Therapies. 2017 October; 22(4):859

6. Garnis P., Nikova A., Birbilis T. Combination of TENS, Lidocaine Injections and Muscle Manipulations as a Therapeutic Method for Cervicogenic Headache - Pilot Study. Maedica (Bucur). 2019 Sep; 14(3): 220- 226.
7. Li C., Zhang Xi-Lu., Ding H., et al. Comparative study on effects of manipulation treatment and transcutaneous electrical nerve stimulation on patients with cervicogenic headache. Journal of Chemical Information and Modeling. $2007 \mathrm{Jul} ; 5(4)$ : 403-6.

8. Penas C., Alonso-Blanco C., Cuadrado M., et al. Spinal Manipulative Therapy in the Management of Cervicogenic Headache. Headache 2005; 45:1260-1270.

9. Kharwandikar P., Shende M. Effectiveness of sub-occipital myofascial release and cervical manipulation in patients with cervicogenic headache. International J. of Healthcare and Biomedical Research. 2019 July; 7(4): 25-32.

10. Tarhan C., Inan L., Karaoglan B., et al. Tens Treatment in Cervicogenic Headache. Physical Medicine 1999; 2 (2): 13-17.

11. Arab A. The Effect of Suboccipital Myofascial Release Technique on Cervical Muscle Strength of Patients with Cervicogenic Headache. PTJ. 2017; 7(1):1928.

How to cite this article: Fursule D, Garg K. Effect of cervical mobilization, transcutaneous electrical stimulation and suboccipital release in cervicogenic headache: a case report. Int $J$ Health Sci Res. 2021; 11(8): 81-85. DOI: https://doi.org/10.52403/ijhsr.20210812 